\title{
Rapid elbow movements in patients with torsion dystonia
}

\author{
W VAN DER KAMP, A BERARDELLI, J C ROTHWELL, P D THOMPSON, B L DAY, \\ C D MARSDEN
}

From the MRC Human Movement and Balance Unit, Institute of Neurology, The National Hospital for Nervous Diseases, Queen Square, London WC1N $3 B G$

SUMMARY Rapid, self paced and self terminated elbow flexion movements were studied in a group of 10 patients with dystonia affecting the arms. The movements were slower and for small amplitude movements, more variable than those recorded in normal subjects. The duration of the first agonist burst was prolonged, even when compared with normal subjects deliberately moving slowly. Cocontraction of agonist and antagonist muscles during ballistic movements was common and may contribute to the bradykinesia. These findings are compared with similar studies of other diseases of the motor system. Unlike many other conditions which also reduce the speed of ballistic voluntary movements, the patients with dystonia in the present study showed a normal symmetry of acceleration and deceleration times. One interpretation of this finding is that aspects of the basic motor programmes are relatively preserved in this condition and account for the surprising retention of motor skills shown by some patients with dystonia.

The abnormal patterns of muscle activation that produce dystonic limb postures in patients with torsion dystonia are usually worse during voluntary movement..$^{1-4}$ Indeed, in its early stages dystonia may be evident only during certain actions such as walking or hand writing. Muscle spasms, jerks and cocontraction of agonist and antagonist muscles distort the limb posture and interrupt the intended sequence of movement. Despite these problems, surprising degrees of motor skill are often retained by patients with advanced torsion dystonia. For example, they may demonstrate great proficiency in the use of hand held communicators, manually operating tools, or as in the case of two of our patients, become accurate marksmen. ${ }^{4}$ It is therefore of some interest to examine the performance of simple motor tasks in patients with upper limb dystonia and the extent to which the above abnormal patterns of muscle activity interfere with the normal simple ballistic movement EMG pattern. Rapid, self-paced and self-terminated movements at a single joint are achieved by a triphasic pattern of

Correspondence to: Professor C D Marsden, University Department of Clinical Neurology, The National Hospital for Nervous Diseases, Queen Square, London WC1N 3BG.

Received 28 January 1989.

Accepted 4 April 1989. muscle activity in the agonist, antagonist and agonist muscles. ${ }^{5-9}$ This pattern has been studied for various disorders of movement ${ }^{10-16}$ and the results from this study in patients with torsion dystonia are compared with those described in patients with other diseases of the basal ganglia, cerebellum, and upper motor neurone pathways.

\section{Patients and methods}

The patients comprised three females and seven males, aged 15 to 79 (mean age 47), all of whom had dystonia affecting one or both upper limbs. Nine patients had primary (idiopathic) torsion dystonia; three of these had segmental dystonia affecting the neck and arms, five had generalised dystonia and one had focal dystonia of an arm. The remaining patient had symptomatic hemidystonia due to an arteriovenous malformation of the contralateral basal ganglia. Six patients were receiving medication (clonazepam, baclofen, benzhexol) at the time of the study. All patients and normal subjects gave informed consent for the studies which were approved by the local ethical committee.

Elbow flexion movements were examined with the subjects seated in a chair and their arm abducted to $90^{\circ}$. The forearm was semipronated and rested on a horizontal manipulandum pivoted about the axis of rotation of the elbow joint. The elbow position was measured by a potentiometer. and displayed on an oscilloscope screen in front of the subject. Electromyographic activity (EMG) was recorded by $\mathrm{Ag}$ / $\mathrm{AgCl}$ surface electrodes placed on the following muscles; 
biceps, triceps, posterior deltoid, pectoralis major and the flexors and extensors of the fingers and wrist in the forearm.

Position, velocity (electronically differentiated from the position) and rectified EMG activity were recorded for each elbow flexion movement with a PDP12 computer, (sampling rate $250 \mathrm{~Hz}$ per channel), using programmes devised by $\mathrm{HB}$ Morton. EMG signals were amplified using a Devices 3160 preamplifier with high and low pass filters set at $80 \mathrm{~Hz}$ and $2.5 \mathrm{kHz}$ (3 dB points) respectively, and a Devices 3120 amplifier.

All subjects were asked to make elbow flexion movements of $15^{\circ}$ and $30^{\circ}$ as fast as they could from a starting angle of $120^{\circ}$. After some practice, 10 to 15 trials for each kind of movement were recorded. Results were compared with values obtained from nine normal subjects aged 29 to 67 (mean age 44). Normal subjects were then asked to perform these movements at roughly half their maximal speed, imitating the velocity of the movements of the dystonic patients.

Each single trial was inspected on the computer display unit. The amplitude, peak velocity of movement and the duration of the first agonist (biceps) EMG burst were measured. The first agonist EMG burst in normal subjects was relatively well defined with clear onset and offset. However, in some patients this was not the case. The duration of the first agonist EMG burst was often difficult to measure since the point at which it terminated was poorly defined. In these patients, the criterion for determining the end of the agonist burst was that the EMG should decline to less than $10 \%$ of the peaksize for at least $20 \mathrm{msec}$. This decrease was expected before the peak velocity of movement. If the first burst of EMG activity continued for more than 50 msec after the peak velocity for any individual movement, then the burst was said to be 'tonic' and not to have a measurable duration. The first antagonist EMG burst was not analysed in these trials. The duration of the movement was measured from the velocity trace and was defined as the time between the onset to the first zero crossing. Movement time was divided into acceleration time (time from the beginning of the movement until the peak velocity) and deceleration time (time from moment of peak velocity until zero velocity). The coefficient of variation for the movement amplitudes were calculated from the mean and standard deviation for each size of movement to give an indication of movement variability. Statistical analysis of the data was performed using unpaired Student's $t$-tests and a mixed model analysis of variance (ANOVA).

\section{Results}

A typical elbow flexion movement through an angle of $30^{\circ}$ performed by a normal subject moving as fast as possible is illustrated in figure 1a. When a patient with dystonia attempted to perform a similar movement of comparable size, as fast as possible (figure 1c), two major differences were evident: i) the movement of the patient was slower than the fast movement of the normal subject and ii) the duration of the first agonist EMG burst (Ag1) in the biceps of the patient was prolonged. The question which arises is whether or not the patients' movements are similar to those of normal

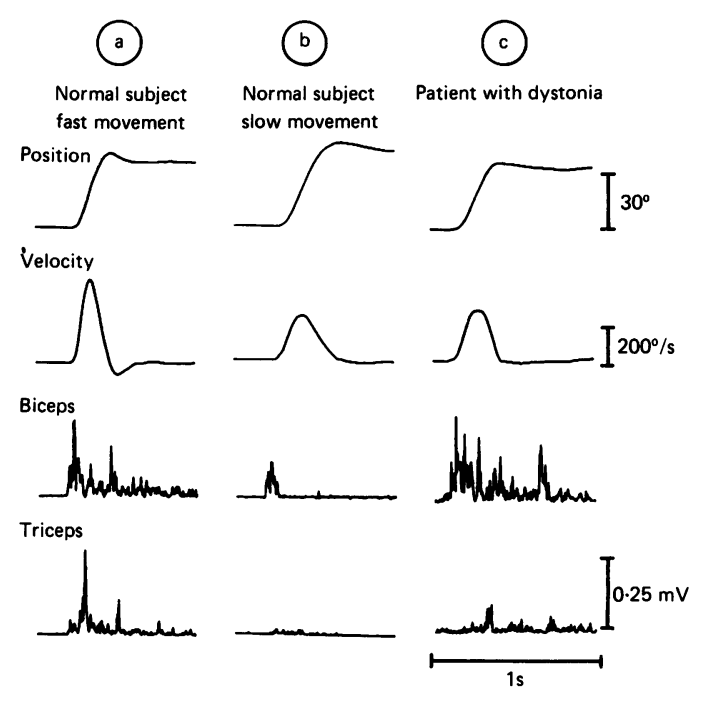

Fig 1 Single trials of a normal subject flexing the elbow through an angle of $30^{\circ}$ as fast as possible (fig la) and at half maximal speed (fig $1 \mathrm{~b}$ ), and of a patient with dystonia (fig 1c). The upper two traces show the position and the velocity of the movement, the lower two traces show the surface rectified EMG recordings of the biceps and triceps muscle. The dystonic patient exhibits slowing of the movement, as well as loss of the characteristic triphasic pattern of EMG activity in biceps and triceps muscle which is evident in the recordings from the normal subject. The deceleration phase of slow moving normals is prolonged.

subjects moving at a comparable velocity (that is, slowly).

To investigate this question we asked the normal subjects to perform a series of movements to the same $15^{\circ}$ and $30^{\circ}$ targets when moving at half their maximal speed. An example of a deliberate slow movement in a normal subject is illustrated in figure $1 \mathrm{~b}$ (the same subject shown in figure 1a). The duration of Agl was still shorter than that of the patient moving at a similar speed. Data were analysed from all subjects and patients for both $15^{\circ}$ and $30^{\circ}$ movements and the following comparisons were made. First, movements of normal subjects moving at their maximal speed were compared with their movements at half their maximal speed. Second, the movements of the patients with dystonia were compared with the slow and fast movements of normal subjects.

\section{Normal subjects}

All nine normal subjects were able to perform the $15^{\circ}$ and $30^{\circ}$ movements with ease, whether moving at maximum speed, or about half that value. There was a tendency for the fast movements to overshoot the target distance before settling down to the final end 


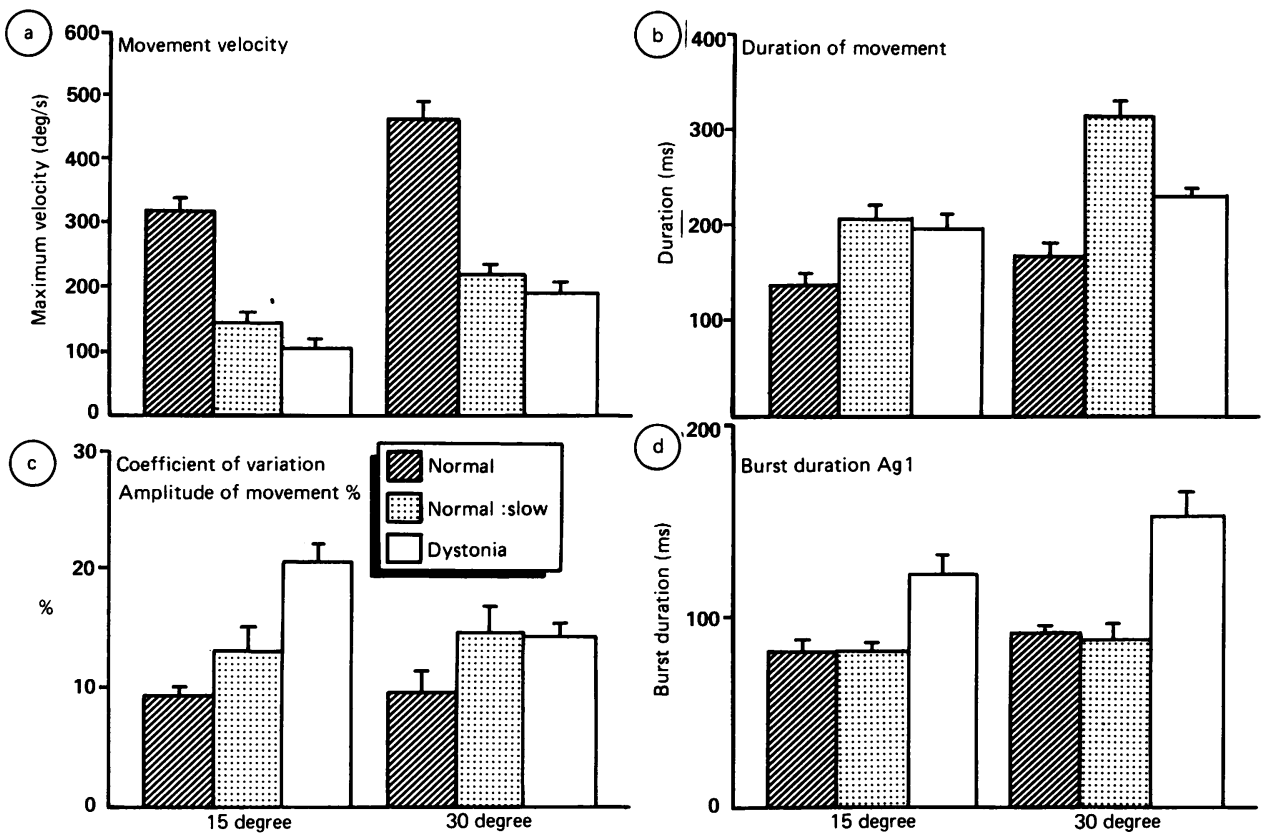

Fig 2 Comparison of various parameters of elbow flexion movements made through an angle of $15^{\circ}$ or $30^{\circ}$ by normal subjects moving as fast as possible and moving at about half their maximal speed and patients with dystonia. Histograms show the mean values and SE. Statistical details are given in the text $(a)$. The maximum velocity of the elbow flexion movements measured as degrees per second. The dystonic patients performed the movements at the same speed as normals moving slowly. (b) The duration of the $15^{\circ}$ and $30^{\circ}$ movements in the normal group and patients with dystonia was similar except when the normal subjects moved slowly (means and SE are shown). (c) The variability from trial to trial is expressed as the coefficient of variation of the movement amplitude. The coefficient of variation for the $15^{\circ}$ movements of the dystonic patients was large (means and SE are shown). (d) The first agonist EMG burst (Ag1) in biceps was measured according to the criteria described in Methods. Although the maximum velocity of the movements of the dystonic patients was similar to that of the slow moving normal subjects (fig 2a), the duration of the Agl EMG bursts was prolonged compared to fast and slow moving normal subjects.

position. The mean (SD) amplitude of fast movements in the $15^{\circ}$ task was $20 \cdot 1^{\circ}\left(2 \cdot 3^{\circ}\right)$, and in the $30^{\circ}$ task was $36^{\circ}\left(2 \cdot 1^{\circ}\right)$. This overshoot was not as evident when the same subjects were moving more slowly (mean [SD] amplitudes of movements were $17^{\circ}\left(1 \cdot 3^{\circ}\right)$, and $32^{\circ}\left(2^{\circ}\right)$ for the $15^{\circ}$ and $30^{\circ}$ tasks respectively). The $30^{\circ}$ movements were performed at about one and a half times the speed of $15^{\circ}$ movements (figure $2 a$ ).

Measurements of acceleration and deceleration times revealed an interesting difference between fast and slow movements. In the fast condition the acceleration time equalled the deceleration time in the $15^{\circ}$ task and was only slightly shorter than the deceleration time in the $30^{\circ}$ movement (table 1). Thus these rapid movements had a roughly bell shaped velocity profile. In the slow task, especially in the $30^{\circ}$ movement, deceleration time exceeded acceleration time by a considerable amount, indicating that subjects tended to glide into the final end position.
The duration of the movement was obviously longer in the slow movements. Comparison of the duration of fast and slow movements in the $15^{\circ}$ and $30^{\circ}$ tasks yielded a significant interaction term in the ANOVA $(F(1,8)=82.98, p<0.05)$, indicating that the movement duration increased more between $15^{\circ}$ and $30^{\circ}$ movements in the slow than in the fast task (figure $2 b$ ). This was a result of the prolonged deceleration phase of the $30^{\circ}$ slow movement. The variability of movement amplitude (expressed as coefficient of variation) was the same whether subjects moved fast or slowly $(F(1,8)=6.15, p>0.05$ ) (figure $2 c)$. The duration of Agl also was the same in both movements $(F(1,8)=0.06, p>0.05)$ (figure 2d).

In normal subjects the first burst of EMG activity in the agonist muscle was followed by a discrete burst of EMG activity in the antagonist muscle. The behaviour of the antagonist burst was similar to that previously described by Marsden, et $a l^{15}$ but has not been 
Table 1 Mean (SE) duration (ms) of acceleration (Acc) $15^{\circ}$ and $30^{\circ}$ in normal subjects making fast and slow movements, and in patients with dystonia

\begin{tabular}{|c|c|c|c|c|}
\hline & \multicolumn{2}{|c|}{$15^{\circ}$ movement } & \multicolumn{2}{|c|}{$30^{\circ}$ movement } \\
\hline & $A c c$ & Dec & $A c c$ & Dec \\
\hline $\begin{array}{l}\text { Normals: } \\
\text { fast } \\
\text { slow } \\
\text { Dystonics }\end{array}$ & $\begin{array}{r}75(7) \\
100(9) \\
116(12)^{*}\end{array}$ & $\begin{array}{c}87(7) \\
134(8)^{*} \\
119(11)^{*}\end{array}$ & $\begin{array}{l}86(7) \\
135(15) \\
130(6)^{*}\end{array}$ & $\begin{array}{l}103(7)^{*} \\
195(5)^{* *} \\
128(5)^{*}\end{array}$ \\
\hline
\end{tabular}

The velocity traces of the movements were used to divide the movement into phases of acceleration and deceleration (see Methods). Asterisks indicate significant differences between acceleration and deceleration times for fast and slow movements in normal subjects $\left({ }^{*} p<0.05 ;{ }^{* *} p<0.01\right)$. Comparisons were also made between the data from patients with dystonia and normal subjects. Patients with dystonia exhibited uniformly longer acceleration and deceleration times than those seen in the fast movements of normal subjects ("p $<0.05$; unpaired Student's $t$ test). However, they were no different from the slow movements of normal subjects $(p>0.05)$, with the exception of the deceleration time of $30^{\circ}$ movements of normal subjects moving slowly. Normal subjects achieved a deliberate slow movement of $30^{\circ}$ amplitude largely by lengthening their deceleration time and gliding to the desired target.

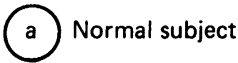

Position

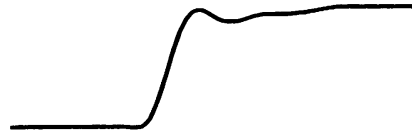

Velocity

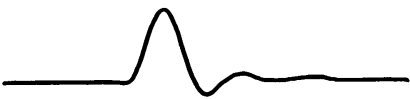

Biceps
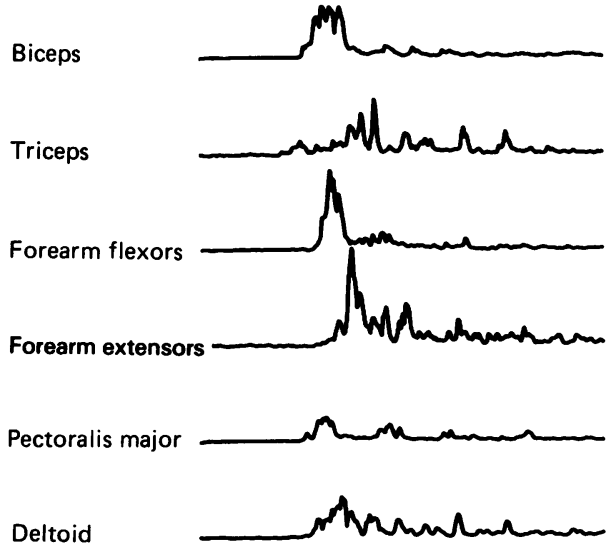
and deceleration (Dec) phases of elbow flexion movements to

analysed in detail here because of the difficulties in measuring data from the patients (see Methods). EMG activity in other muscles of the arm (forearm flexors, extensors, pectoralis major, deltoid) showed an alternating pattern of EMG activity. The forearm flexors, pectoralis major and posterior deltoid were activated at the same time as the biceps; the forearm extensors were activated later or at the same time as the triceps (figure 3a).

\section{Patients with dystonia}

Nine of the 10 patients were able to perform the required elbow flexion movements and flex their elbow to approach the desired target. The mean (SD) amplitudes of movement in the patient group were slightly smaller $\left[15^{\circ}\left(4^{\circ}\right)\right.$ for the $15^{\circ}$ target and $29^{\circ}\left(5^{\circ}\right)$ for the $30^{\circ}$ target] than those achieved by the normal subjects moving slowly $(F(1,16)=4.76 ; p<0.05)$ or as fast as possible $(\mathrm{F}(1,16)=23.66 ; \mathrm{p}<0.001)$.

The fastest elbow flexion movements of the patients with dystonia were slower than the fastest movements of normal subjects $(F(1,16)=68.96, p<0.001)$
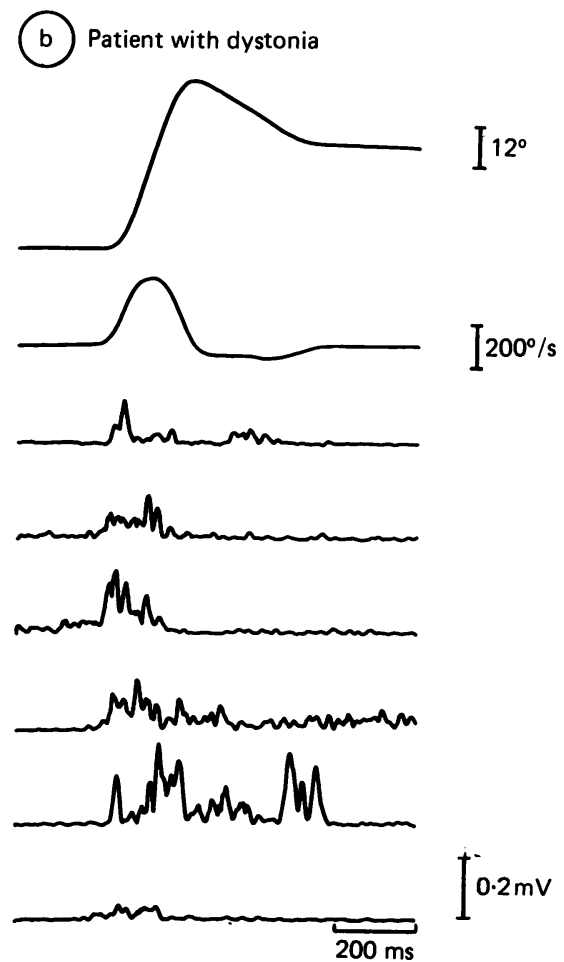

Fig 3 Single trials of an elbow flexion movement to a $30^{\circ}$ target in a normal-subject (a) and a patient with dystonia (b). The top two traces show the position and velocity records while the remainder show rectified EMG recordings from biceps, triceps, forearm flexors and extensors, pectoralis major and deltoid muscles. The burst duration of the agonist muscle (biceps) in the elbow flexion movement is prolonged in the patient with dystonia, and the timing of agonist and antagonist muscle contractions is inappropriate for the movement. Note also the prolonged activation of pectoralis major (overflow) and cocontraction of the forearm and shoulder muscles. 
(figure 2a). Although the velocity of $30^{\circ}$ movements was greater than that of $15^{\circ}$ movements in both groups, the increment in velocity was much smaller in the patients, giving rise to a significant interaction in the ANOVA $(F(1,16)=9.04, p<0.05)$. In contrast, the slow movements of the normal subjects were executed at the same speed as the fastest movements of the patients $(F(1,16)=0.34, p>0.05)$ (see figure 2a).

Acceleration and deceleration times were the same as in the slow normal group except for the $30^{\circ}$ movement. When normals performed a slow $30^{\circ}$ movement, the deceleration time was much longer than in the patients (table 1). This was because when the normal group moved slowly over a long distance, they tended to glide into the final target whereas the patients did not. The result of this can be seen in the comparison of movement durations. The duration of $15^{\circ}$ movements was the same in the patients as in the slow normal group, but in the $30^{\circ}$ task, the patients took less time than the normal group moving slowly (figure 2b). This difference gives rise to the interaction term in the comparison of movement duration $(\mathrm{F}(1,16)=29, \mathrm{p}<0.001)$.

The amplitude of attempted $15^{\circ}$ movements was more variable from trial to trial in the patients than in normal subjects, whether the latter were moving slow or fast (Student's $t$ test $\mathrm{p}<0.01$ ) (figure 2c). The variability of $30^{\circ}$ movements was the same in all groups (Student's $t$ test $p>0.05$ ). The duration of the first agonist EMG burst was longer in the patients for both $15^{\circ}$ and $30^{\circ}$ tasks whether compared with normal subjects moving fast $(\mathrm{F}(1,16)=16.43, \mathrm{p}<0.001)$, or slow $(\mathrm{F}(1,16)=16 \cdot 15, \mathrm{p}<0.001)$ (figure $2 \mathrm{~d}$ ).

Several patterns of antagonist EMG activity were observed. These varied from a normal appearance to cocontraction often with prolonged bursts or tonic EMG activity without recognisable bursts. The patterns of EMG activity in the forearm flexors and extensors, and in the pectoralis major and posterior deltoid muscles, also varied from relatively normal patterns, with appropriate alternating activity in these agonist-antagonist muscle pairs, to various patterns of prolonged bursts in these muscle groups. An example of a movement in a patient with arm dystonia is shown in figure $3 \mathrm{~b}$. Cocontraction of the agonist (biceps) and antagonist (triceps) muscles can be seen, and there abnormal activation of the pectoralis major muscle (overflow).

Among the 10 patients studied, the findings in the patient with symptomatic hemidystonia were similar to those in patients with idiopathic dystonia. One severely affected individual with generalised idiopathic torsion dystonia was unable to perform the elbow flexion task. On attempting to flex the elbow he could only produce movements of $40-60^{\circ}$ and was unable to make movements of smaller amplitude. The accompanying EMG bursts were greatly prolonged and frequently had to be classified as tonic (see Methods), while the maximum velocity was $232^{\circ}$ (SD $40^{\circ}$ ) per second was even slower than the other patients.

\section{Discussion}

Rapid self paced and self terminated movements at a single joint have now been studied in patients with a wide variety of disorders of voluntary and involuntary movement. ${ }^{10-16}$ Our results extend this analysis to patients with torsion dystonia. Their elbow flexion movements differed from normal in the following ways: 1) the peak velocity was slower, 2) the extent of movement was more variable for the small, but not the large amplitude task, 3) the duration of the first burst of agonist EMG activity was prolonged even when compared with the normal group who had been purposely instructed to move slowly, at a similar speed to the patients, 4) there often was cocontraction of the antagonist muscle.

The movements of dystonic patients, however, were normal in one respect. The acceleration and deceleration times were approximately equal indicating that the velocity profile was bell shaped. ${ }^{17}$ The same profile was seen in the fast movements of the normal subjects, although it became skewed towards longer deceleration times when the same normal individuals were instructed to move slowly, particularly in the $30^{\circ}$ task. The bell shaped velocity profile of the dystonic patients indicates that despite the abnormal pattern of EMG activity, at least this aspect of the ballistic motor programme appears to remain intact.

Table 2 compares the deficits in the present dystonic patients with those reported in other neurological conditions. Interestingly the movements of all groups are slower than normal, perhaps indicating that rapid movements of normal subjects are optimal and that their velocity cannot be exceeded in any pathological state. Although all groups move more slowly than normal, combinations of increased movement variability, asymmetric velocity profile and prolonged and/or cocontracting EMG activity distinguish between several of the groups. Not surprisingly, the voluntary movements of patients with dystonia appear very similar to those of patients with athetosis ${ }^{12}$ and patients with Huntington's disease, ${ }^{14}$ although there is a lack of data on the velocity profiles of the latter condition. Despite the clinical differences between the involuntary sustained muscle spasms which produce relatively fixed postures in dystonia (and athetosis) and the continual flow of movement in chorea, the control of simple voluntary movements exhibits similarities in the two conditions. The motor symptoms of dystonia, athetosis and chorea are 
Table 2 Comparison of ballistic movements in patients with different disorders of movement

\begin{tabular}{|c|c|c|c|c|c|c|}
\hline & $\begin{array}{l}\text { Upper motor } \\
\text { neurone }\end{array}$ & $\begin{array}{l}\text { Cerebellar } \\
\text { ataxia }\end{array}$ & $\begin{array}{l}\text { Parkinson's } \\
\text { disease }\end{array}$ & $\begin{array}{l}\text { Huntington's } \\
\text { disease }\end{array}$ & Athetosis & Dystonia \\
\hline $\begin{array}{l}\text { Amplitude variability } \\
\text { Peak velocity } \\
\text { Velocity profile } \\
\text { Duration Ag } 1 \\
\text { Ag/Ant pattern }\end{array}$ & $\begin{array}{l}\text { NK } \\
\text { Slow } \\
\text { NK } \\
\text { Prolonged } \\
\text { N }\end{array}$ & $\begin{array}{l}\text { *Increased } \\
\text { Slow } \\
\text { *Asymm } \\
\text { Prolonged } \\
\text { Variable }\end{array}$ & $\begin{array}{l}\mathbf{N} \\
\text { Slow } \\
+\mathbf{N} \\
\mathbf{N} \\
\mathbf{N}\end{array}$ & $\begin{array}{l}\text { Increased } \\
\text { Slow } \\
\text { NK } \\
\text { Prolonged } \\
\text { Cocontracting }\end{array}$ & $\begin{array}{l}\text { Increased } \\
\text { Slow } \\
\text { NK } \\
\text { Prolonged } \\
\text { Cocontracting }\end{array}$ & $\begin{array}{l}\text { Increased ( } 15^{\circ} \text { only) } \\
\text { Slow } \\
\text { N } \\
\text { Prolonged } \\
\text { Cocontracting }\end{array}$ \\
\hline
\end{tabular}

All were self-paced and self-terminated elbow or wrist flexion movements, made as rapidly as possible. Measured mechanical parameters are (i) variability in peak amplitude from trial to trial; (ii) average peak velocity; (iii) velocity profile with relative duration of acceleration and deceleration phases of the movement. Measured EMG parameters are the average duration of the first burst of agonist (flexor) EMG (Ag 1 ) and the approximate time relations of agonist and antagonist EMG activity ( $\mathrm{Ag} / \mathrm{Ant}$ pattern). Descriptions refer to comparison with data from normal subjects executing movements of the same extent under the same instructions. Results taken from this paper and previously published work of ourselves and other authors. ${ }^{11-16}$

NK $=$ not known; $\mathbf{N}=$ normal; Asymm = asymmetrical profile. ${ }^{*}$ Mark Hallett, personal communication. †Calculated from a reexamination of the data of Berardelli et al. ${ }^{13}$

thought to be due to abnormal function of the basal ganglia, so the common deficits of voluntary movement in all three conditions may reflect disruption of the normal basal ganglia contribution to rapid simple movements. The prolonged, excessive and variable patterns of voluntary muscle activity seen in patients with dystonia, athetosis and chorea may indicate, as Hallet $\mathrm{t}^{11}$ suggests, that the basal ganglia play a role in selectively activating only those muscles appropriate for a particular task and inhibiting those which are not required. Other physiological studies in dystonia also point to excessive muscle activation and/or lack of normal inhibition of muscle activity. The R2 component of the blink reflex is prolonged and its recovery curve hyperexcitable in patients with blepharospasm ${ }^{18}$; there is a reduction in the amount of Ia presynaptic inhibition in patients with dystonia affecting the arm; ${ }^{419}$ the duration of the long-latency stretch reflex in the wrist flexor muscles is prolonged. ${ }^{20}$

The EMG pattern responsible for the slow movements of the patients with Parkinson's disease is quite different ${ }^{1113}$ from that of dystonic, athetoid and choieic patients. The agonist and antagonist bursts are timed correctly and appropriate muscles are activated in Parkinson's disease, but the EMG bursts are smaller than necessary to produce the same rapid movements shown in the normal group. Why then does this classic disease of the basal ganglia produce different abnormalities to those seen in dystonia, athetosis, and chorea? We have suggested previously ${ }^{14}$ that the basal ganglia circuitry as presently conceived (for example 21) allows for the possible coexistence of bradykinesia and hyperkinesia. In essence there may be at least two circuits directly concerned with movement within the basal ganglia; abnormalities of the one involved with the initiation of a voluntary movement may result in bradykinesia; abnormalities in the other, which may be involved in minimising unwanted or extraneous movement could lead to dyskinesias. One or both circuits may be differentially affected in different disease states. Thus, in Parkinson's disease, loss of the dopaminergic nigrostriatal input may affect the former pathway whereas both might be affected in dystonia, athetosis and chorea.

Finally, why are the movements of patients with dystonia slower than normal subjects? The patients are not weak and their EMG bursts are not shorter than normal. If we assume that the EMG-force relation of the muscles is normal, three other possibilities emerge: 1) peak velocities may be reduced because the agonist EMG burst is prolonged; a large agonist burst capable of producing normal peak velocities, if excessively prolonged would take the limb beyond theo target point. To prevent overshoot, the burst would have to be selected to be smaller and, hence, peak velocity inevitably would be reduced; 2) action of the agonist muscle might be opposed by inappropriate cocontracting activity of the antagonist; 3 ) there may be a primary difficulty in producing maximum bursts of phasic muscle force even though voluntary strength is intact. In the most rapid contractions, motoneurones discharge phasically at rates of up to $200 \mathrm{~Hz}$. Presumably a very large descending input is needed to produce these rates and the pathology of dystonia may make this impossible; the $30-40 \mathrm{~Hz}$ rate for a normal fused voluntary contraction might be achieved relatively easily, but the resulting contraction would be slower.

In conclusion, these experiments have shown that rapid, self-paced and self-terminated movements of patients with dystonia are slower and more variable than normal. Deficits in the duration of the first agonist EMG burst and in control of antagonist and synergist muscles are similar to those seen in patients with Huntington's disease or athetosis. Such deficits may reflect a common contribution of the basal ganglia to the control of ballistic movements, perhaps involving the grading of EMG agonist burst size and duration and the selection and timing of appropriate agonist and antagonist muscle activity. 
We thank Mr HC Bertoya and Mr R Bedlington for their invaluable assistance in constructing and maintaining much of the equipment used in these experiments. The work was funded by the Medical Research Council, and the research funds of the Bethlem Royal and Maudsley Hospitals.

\section{References}

1 Hoeffer PFA, Putnam TJ. Action potentials of muscles in athetosis and Sydenham's chorea. Arch Neurol Psychiat 1940;44:517-31.

2 Herz E. Dystonia I. Historical review: analysis of dystonic symptoms and physiological mechanisms. Arch Neurol Psychiat 1944;51:305-18.

3 Yanigisawa N, Goto A. Dystonia musculorum deformans. $J$ Neurol Sci 1971;13:39-65.

4 Rothwell JC, Obeso JA, Day BL, Marsden CD. Pathophysiology of dystonias. Adv Neurol 1983;39:851-63.

5 Hallett M, Shahani BT, Young RR. EMG analysis of stereotyped voluntary movements in man. J Neurol Neurosurg Psychiatry 1975;38:1154-62.

6 Angel RW. Electromyography during voluntary movement. The two burst pattern. Electroenceph clin Neurophysiol 1975;36: 433-8.

7 Hallett M, Marsden CD. Ballistic flexion movements of the human thumb. J Physiol (Lond) 1979;294:33-50.

8 Brown SH, Cooke JD. Amplitude and instruction dependent modulation of movement related EMG activity in humans. J Physiol (Lond) 1981;316:97-107.

9 Berardelli A, Rothwell JC, Day BL, Kachi T, Marsden CD. Duration of the first agonist EMG burst in ballistic arm movements. Brain Res 1984;304:183-7.

10 Hallett M, Shahani BT, Young RR. EMG analysis of patients with cerbellar deficits. J Neurol Neurosurg Psychiatry 1975;38. $1163-9$.

11 Hallett $M$, Khoshbin S. A physiological mechanism of bradykinesia. Brain 1980;103:301-14.

12 Hallett M, Alvarez N. Attempted rapid elbow movements in patients with athetosis. $J$ Neurol Neurosurg Psychiatry 1983; 46:745-50.

13 Berardelli A, Dick JPR, Rothwell JC, Day BL, Marsden CD. Scaling of the size of the first agonist EMG burst during rapid wrist movement in patients with Parkinson's disease. $J$ Neurol Neurosurg Psychiatry 1986;49:1273-9.

14 Thompson PD, Berardelli A, Rothwell JC, et al. The coexistence of bradykinesia and chorea in Huntington's disease and its implications for theories of the basal ganglia and control of movement. Brain 1988;111:223-44.

15 Hallett M. Ballistic elbow flexion movements in patients with amyotrophic lateral scoliosis. J Neurol Neurosurg Psychiatry 1979;42:232-7.

16 Fagioli S, Berardelli A, Hallett M, Accornero N, Manfredi M. The first agonist and antagonist burst in patients with an upper motor neuron syndrome. Movement Disorders 1988;3:126-32.

17 Ostry DJ, Cooke JD, Munhall KG. Velocity curves of human arm and speech movements. Exp Brain Res 1987;68:37-46.

18 Berardelli A, Rothwell JC, Day BL, Marsden CD. Pathophysiology of blepharospasm and oromandibular dystonia. Brain 1985;108:593-608.

19 Nakashima K, Rothwell JC, Day BL, Shannon K, Thompson PD, Marsden CD. Reciprocal inhibition between forearm muscles in patients with writer's cramp and other occupational dystonias and patients with hemiparesis due to stroke. Brain 1989;112: 681-97.

20 Tatton WG, Bedingham W, Verrier MC, Blair RAG. Characteristic alterations in responses to imposed wrist displacement in parkinsonian rigidity and dystonia musculorum deformans. Can J Neurol Sci 1984;11:281-7.

21 Penney JB, Young AB. Striatal inhomogeneities and basal ganglia function. Movement Disorders 1986;1:3-15. 